\title{
Clinical Experience of Using Cortical Auditory Evoked Potentials in the Treatment of Infant Hearing Loss in Australia
}

\author{
Simone Punch, M.Sc., ${ }^{1}$ Bram Van Dun, Ph.D., ${ }^{2,3}$ Alison King, M.Sc., 1 \\ Lyndal Carter, M.Sc., ${ }^{2,3}$ and Wendy Pearce, M.Sc. ${ }^{1}$
}

\section{ABSTRACT}

This article presents the clinical protocol that is currently being used within Australian Hearing for infant hearing aid evaluation using cortical auditory evoked potentials (CAEPs). CAEP testing is performed in the free field at two stimulus levels $(65 \mathrm{~dB}$ sound pressure level [SPL], followed by 55 or $75 \mathrm{~dB}$ SPL) using three brief frequencydistinct speech sounds $/ \mathrm{m} /, \mathrm{g} /$, and $/ \mathrm{t} /$, within a standard audiological appointment of up to 90 minutes. CAEP results are used to check or guide modifications of hearing aid fittings or to confirm unaided hearing capability. A retrospective review of 83 client files evaluated whether clinical practice aligned with the clinical protocol. It showed that most children could be assessed as part of their initial fitting program when they were identified as a priority for CAEP testing. Aided CAEPs were most commonly assessed within 8 weeks of the fitting. A survey of 32 pediatric audiologists provided information about their perception of cortical testing at Australian Hearing. The results indicated that clinical CAEP testing influenced audiologists' approach to rehabilitation and was well received by parents and that they were satisfied with the technique. Three case studies were selected to illustrate how CAEP testing can be used in a clinical environment. Overall, CAEP testing has been effectively integrated into the infant fitting program.

KEYWORDS: Cortical auditory evoked potential, hearing, hearing aid, infant

${ }^{1}$ Australian Hearing, Sydney, Australia; ${ }^{2}$ The HEARing CRC, Melbourne, Australia; ${ }^{3}$ National Acoustic Laboratories, Sydney, Australia.

Address for correspondence: Simone Punch, M.Sc., Australian Hearing, Australian Hearing Hub, Level 5, 16 University Avenue, Macquarie University NSW 2109, Australia (e-mail: simone.punch@hearing.com.au).
The Use of Cortical Auditory Evoked Potentials in Diagnosis and Treatment of Hearing Disorders; Guest Editor, Bram Van Dun, Ph.D.

Semin Hear 2016;37:36-52. Copyright (C) 2016 by Thieme Medical Publishers, Inc., 333 Seventh Avenue, New York, NY 10001, USA. Tel: +1(212) 584-4662. DOI: http://dx.doi.org/10.1055/s-0035-1570331. ISSN 0734-0451. 
Learning Outcomes: As a result of this activity, the participant will be able to describe the clinical protocols implemented at Australian Hearing for the use of cortical auditory evoked potential testing for hearing aid fitting and evaluation in young children.

Australia has national coverage of universal newborn hearing screening (UNHS), which is now operating in all states and territories under a National Framework incorporating evidence-based standards of practice. ${ }^{1}$ Babies diagnosed with a hearing loss are followed up by one of the 25 specialized Australian Hearing infant hearing fitting centers. Australian Hearing is a government-funded organization that provides audiological services including hearing aid and cochlear implant (CI) support to close to 21,000 young Australians (aged under 26 years) at no cost to families, except for a small annual device maintenance fee. For the 2012 birth year, 386 infants were fitted with their first hearing aids before 6 months of age. This figure is considered to represent all infants fitted in 2012 in Australia, because Australian Hearing is the sole provider of this service.

The evaluation of hearing aid fittings for infants has long proved difficult for audiologists, due to the challenges of observing behavioral responses to sound in very young infants. Cortical auditory evoked potentials (CAEPs) are applicable to infant hearing aid evaluation and also may be regarded as the auditory evoked response technique most suited to assessing the audibility of hearing aid-amplified speech. ${ }^{2}$ Although it is feasible to use evoked potentials such as the auditory brainstem response (ABR) and auditory steady-state response (ASSR) to assess hearing aid fitting when behavioral measures cannot be employed, recording CAEPs for this purpose has several advantages over recording early latency electrophysiologic measures. First, the acoustic features that are relevant for speech detection and perception can be presented as stimuli, ${ }^{3}$ which are handled reasonably well by a hearing aid. Second, the integrity of the response pathway through to the cortex can be assessed, ${ }^{4}$ therefore the presence and absence of CAEPs correlates better with perception. ${ }^{5}$ Third, in some cases of auditory neuropathy spectrum disorder (ANSD), a CAEP may be observed even when an ABR is absent. ${ }^{6,7}$ Fourth, CAEPs are proven to be reliably recorded in young awake infants with normal hearing when stimuli are presented at suprathreshold levels. ${ }^{8}$ In children with hearing impairment, more care has to be taken, however, when interpreting absent CAEPs because more than $20 \%$ of the children in this group did not show detectable CAEPs, even when the sounds were audible. ${ }^{9-11}$

Research involving the use of CAEPs for hearing aid evaluation at the National Acoustic Laboratories (NAL), and around the world, has made it possible to record CAEPs in a clinical setting. Earlier work has shown that CAEPs in aided children are larger than in unaided children and adults. ${ }^{2,12,13}$ Research influenced by NAL's earlier work in this area has shown that short speech sounds with low-, mid-, and highfrequency content, presented in the free field, can be used to evaluate audibility in unaided and aided clients. 5,6,10,14-22 Golding et al showed a significant correlation between CAEPs and parent observations of functional performance systematically recorded using the Parents' Evaluation of Aural/Oral Performance of Children $(\mathrm{PEACH}) .{ }^{19}$ Based on this information and experience, a clinical device (HEARLab Frye Electronics, Tigard, Oregon) was developed.

CAEP testing using HEARLab was introduced into the Australian Hearing infant clinical pathways during 2011. Infant hearing aid fittings are now systematically evaluated, when clinically appropriate, using CAEP assessment. This article describes the clinical protocol that is currently being used within Australian Hearing. As the scientific value of CAEPs for (basic) hearing aid fitting evaluation is still under debate, ${ }^{2,23-33}$ there are limited published data that investigate the feasibility of using this test in a clinical setting. To address this gap in knowledge, Australian Hearing reviewed over 80 client files to evaluate whether clinical practice aligned with the clinical protocol. A survey of pediatric audiologists regarding the use of CAEPs in the clinical setting also was conducted to collect information about their perception of cortical testing at Australian 
Hearing. Three case studies are also provided, demonstrating the clinical use of CAEPs in infant hearing aid fitting for the three major reasons CAEP testing is conducted in the Australian Hearing clinic.

\section{CLINICAL INFANT HEARING AID FITTING EVALUATION USING CORTICAL AUDITORY EVOKED POTENTIALS IN AUSTRALIA}

The protocol described in this section is nationally implemented by Australian Hearing. ${ }^{34}$ The protocol is based on a comprehensive review of international research into CAEPs and the clinical experience gained during the clinical development and evaluation of the HEARLab system at NAL. 2,3,5,6,9,10,13,18-20,32,35-39 Aided CAEP assessment now forms part of the clinical pathway for children and other clients whose hearing aid prescription has been based on an audiogram estimated from evoked potential tests (ABR, ASSR). It was introduced into Australian Hearing's clinical program for the following reasons.

- CAEP assessment possibly may add to the information obtained using more traditional assessment techniques, such as behavioral observation audiometry, as it provides a systematic and objective means of indicating with some level of confidence that low-, mid-, and high-frequency emphasis speech sounds are audible to the aided child. ${ }^{9-11}$

- For many children, particularly those with moderate to severe hearing loss, CAEPs can be used to demonstrate aided benefit to parents in a tangible way, ${ }^{9-11,13,22}$ which may encourage consistent hearing aid use.

- Where some CAEPs are absent, the pattern of results (considering both stimulus type and presentation level) may give a general guide to adjusting a child's hearing aid, to achieve audibility for speech at conversational levels. ${ }^{2,9-11,16,22,32}$ It needs to be noted that absent CAEPs not necessarily indicate a (amplified) sound is inaudible to the child with hearing impairment. ${ }^{9-11}$

- Where CAEP responses are repeatedly absent, even after increases to the gain-frequency response of a child's hearing aids, results may help parents and professionals in considering moving toward CI evaluation, when combined with other audiological information.

\section{HOW ARE CORTICAL AUDITORY EVOKED POTENTIALS CURRENTLY IMPLEMENTED FOR HEARING AID FITTING EVALUATION IN AUSTRALIAN HEARING?}

- CAEPs form part of the initial fitting program for all, ${ }^{34}$ including clients whose hearing aid fitting has been based upon an audiogram estimated from evoked potentials (tone burst $\mathrm{ABR}$ or ASSR), most commonly infants referred from UNHS, and children who have ANSD. Both aided and unaided CAEPs are used in ANSD management. Research into CAEPs with children with ANSD has shown that where CAEPs are present at low stimulus levels, subsequent behavioral testing will likely show hearing at normal levels or a mild hearing loss. 6,7

The test is also applicable for clients of any age who have additional disabilities that have led to a hearing aid fitting based upon minimal behavioral information or that have made it difficult to evaluate the benefit of their hearing aid fitting, and clients who are being considered for cochlear implantation due to poor speech perception performance or delayed speech/language development.

CAEP testing is not a priority for children who have a unilateral hearing loss or a threefrequency average hearing loss that is estimated to be less than $45 \mathrm{~dB}$ hearing level (HL). The value of $45 \mathrm{~dB}$ HL was chosen based on the assumption that a hearing loss of $45 \mathrm{~dB}$ or better will have a $10 \mathrm{~dB}$ sensation level to a $55 \mathrm{~dB}$ sound pressure level (SPL) speech sound. This will likely result in a CAEP response when the person is unaided. ${ }^{9}$ As a result, the aided and unaided conditions are difficult to compare.

Fig. 1 shows the Australian Hearing pathway for the initial fitting and evaluation of infants. ${ }^{34}$ CAEP evaluation is usually conducted after the first follow-up appointment, 


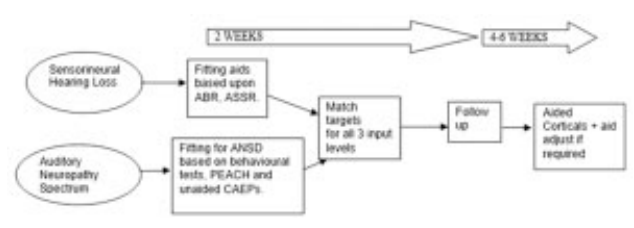

Figure 1 Clinical pathway for aided CAEP assessment within Australian Hearing pediatric program. Abbreviations: ABR, auditory brainstem response; ANSD, auditory neuropathy spectrum disorder; ASSR, auditory steady-state response; CAEP, cortical auditory evoked potential; PEACH, Parents' Evaluation of Aural/Oral Performance of Children.

which allows the clinician to provide support and encouragement to the family in their management of their child's hearing aids before embarking upon the evaluation. Hearing aids are fitted according to the national protocol for pediatric amplification in Australia, ${ }^{36}$ recently updated to include the use of NAL-NL: $\mathrm{Na}^{-}$ tional Acoustic Laboratories - Non Linear prescriptive targets derived from a behavioral audiogram that has been either estimated from evoked potential tests or measured using a behavioral procedure. For children diagnosed with ANSD, a battery of measures, including behavioral assessments and functional questionnaires, is used to derive the estimated audiogram. $^{40}$

A review appointment is conducted within 2 weeks of the fitting. This appointment is devoted mainly to providing support and encouragement to the family in their management of their child's hearing aids. A further appointment within 4 to 6 weeks of the first follow-up is then arranged to evaluate the effectiveness of the hearing aid fitting. This second follow-up appointment is an appropriate time for aided CAEP evaluation. This typically means that aided CAEP evaluation is completed within 8 weeks of the initial hearing aid fitting.

The first CAEP assessment is performed aided, unless the child is diagnosed with ANSD. Three brief speech stimuli (30 milliseconds $/ \mathrm{m} /$, 21 milliseconds $/ \mathrm{g} /$, and 30 milliseconds / $\mathrm{t} /$ with dominant frequencies at 250 , 1,250 , and $3,250 \mathrm{~Hz}$, respectively) are presented in the free field through a loudspeaker at 55,65 , or $75 \mathrm{~dB}$ SPL, as described in Van Dun et al. ${ }^{22}$ If the infant is unsettled and it is likely that not all planned stimuli and levels can be tested, the audiologist prioritizes stimulus presentations according to the assumed individual audiogram configuration and other clinical considerations such as degree of hearing loss and aided speech-o-gram. ${ }^{34}$ The speech-ogram is a visual display of the long term root mean square spectrum and dynamic range of amplified speech superimposed on the child's hearing thresholds. ${ }^{41}$ Audiologists use this as a guide to the expected audibility of amplified speech, and thus expected CAEPs, at chosen decibels of SPL levels. For example, if the main concern is aided audibility at the higher frequencies, the clinician might opt to begin testing with the /t/ sound only. Fig. 2 shows the recommended flowchart for interpretation of each separate speech stimulus. ${ }^{34}$ In summary:

- If a CAEP is obtained at 55 and $65 \mathrm{~dB}$ SPL, no change to the client's estimated hearing thresholds (and hence the hearing aid frequency response) is required.

- If a CAEP is present at $65 \mathrm{~dB}$ SPL but not at $55 \mathrm{~dB}$ SPL (and detection would be expected based upon the NAL-NL2 aided speech-o-gram for a $55 \mathrm{~dB}$ SPL speech signal), the revised hearing thresholds are assumed to be 1 standard deviation (SD), or $10 \mathrm{~dB},{ }^{36,42}$ poorer than the original estimated thresholds at the corresponding frequency range.

- If a CAEP is present at $75 \mathrm{~dB}$ SPL but not at $65 \mathrm{~dB}$ SPL, revised hearing thresholds are assumed to be $1 \mathrm{SD}$, or $10 \mathrm{~dB}$, poorer than the original estimated thresholds at the corresponding frequency range.

- If no CAEPs are present at 65 or $75 \mathrm{~dB}$ SPL, revised hearing thresholds are assumed to be $1.5 \mathrm{SD}$, or $15 \mathrm{~dB}$, poorer than the original estimated threshold at the corresponding frequency range.

If after hearing aid adjustment (based on the revised estimated hearing thresholds determined above) no CAEPs are present, the $75 \mathrm{~dB}$ SPL input aided speech-o-gram, based on the latest estimated audiogram, is inspected. If the speech-o-gram suggests that no detection of the speech spectrum in the frequency range under consideration is expected, the result is consistent with expectations. If no CAEPs are present 


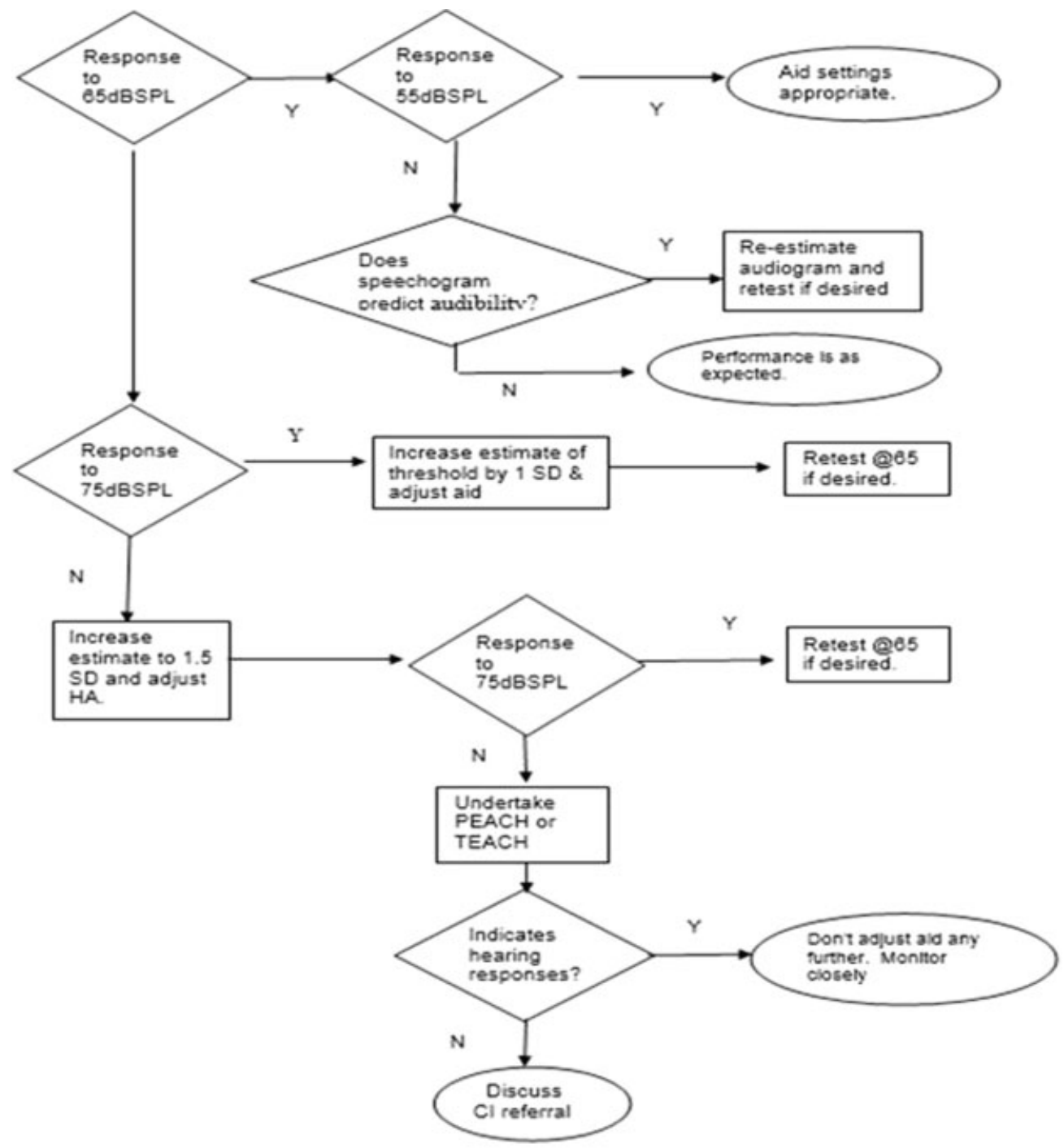

Figure 2 Aid adjustment per speech stimulus. Abbreviations: $\mathrm{Cl}$, cochlear implant; $\mathrm{HA}$, hearing aid; $\mathrm{PEACH}$, Parents' Evaluation of Aural/Oral Performance of Children; SD, standard deviation; SPL, sound pressure level: TEACH, Teachers' Evaluation of Aural/Oral Performance of Children.

and the audiogram has already been reestimated to be $1.5 \mathrm{SD}$ poorer than the average, the $\mathrm{PEACH}$ is used to determine whether there are any behavioral responses to sound in the child's real-life environment. ${ }^{43,44}$ This helps to determine whether the child is one of a limited number whose aided CAEPs do not reflect their hearing capacity. It is important to note that CAEPs are not always detected in some children, even when the (aided or unaided) sound is audible. ${ }^{9-11}$ This can be caused by straightforward reasons like the presence of middle ear disease (which always should be checked prior to performing CAEPs), but also unknown reasons that are still subject to research (e.g., maturation, stimulus rate, or speech discrimination ability). A consistent CAEP absence for multiple speech sounds, levels, and visits, however, might be an indication that closer follow-up is warranted. ${ }^{9} \mathrm{CAEP}$ results combined with $\mathrm{PEACH}$ observations also are used to determine whether referral for $\mathrm{CI}$ candidacy evaluation is appropriate.

Once the audiogram has been reestimated and the hearing aid adjusted to the new NALNL2 prescription targets for that hearing loss, ${ }^{36}$ 
CAEP assessment can be repeated to determine whether aid adjustments have improved audibility of the speech spectrum.

\section{ANALYSIS OF THE CLINICAL APPLICATION OF CORTICAL AUDITORY EVOKED POTENTIALS IN THE MANAGEMENT OF INFANTS WITH HEARING LOSS}

The routine use of CAEPs to evaluate infant hearing aid fittings is relatively new in the field of audiology in general and within Australian Hearing's program in particular. As part of Australian Hearing's ongoing quality assurance program, the clinical application of CAEPs within infant fitting programs was reviewed using a national sample of clinical cases. This review and survey aimed to determine the uptake of the new procedure, the compliance with protocols, the feasibility of using the CAEP test in daily clinical practice, and the influence of CAEPs on clinical management of infants with hearing loss.

\section{METHODS}

As part of Australian Hearing's quality review process, a file review conducted by the first author in the latter part of 2013 examined 87 files of all infants fitted with their first hearing aids between October and December 2012, before the age of 6 months. The file review considered the number of children for whom CAEP testing was recommended by the Australian Hearing protocols, the number actually tested, the number of children who were not tested despite indications to do so, and the reasons why testing was not completed. The review also investigated the timing of the CAEP assessment within the child's program and how often individual children were assessed.

Forty-eight Australian Hearing pediatric audiologists were invited to participate to an online survey regarding their experience with the training program, the stimuli and presentation levels typically used during a clinical appointment, clinical decision making, and their opinion of the value of using CAEPs in their clinical management of infants with hearing loss. Participants for the audiologist survey were drawn from the e-mail distribution list used for peer support within Australian Hearing. A reminder was sent after 2 months if the survey was not returned. Thirty-two clinicians responded to the survey at the end of 2014. Surveys were anonymously completed online using SurveyMonkey. This survey was conducted with the approval of the Australian Hearing Human Research Ethics Committee and conformed to National Health \& Medical Research Committee guidelines.

\section{RESULTS}

\section{File Reviews}

Most children whose files were audited had completed their initial fitting program at the time of the review. The initial infant fitting program usually includes a first appointment, a fitting, an initial follow-up appointment, and at least one subsequent follow-up. Over the four appointments, activities such as discussion of amplification choices, earmold impressions, hearing aid fitting, and evaluation of aided performance are performed. Once this program is completed, children commence subsequent hearing and communication improvement programs that focus on specific needs and goals.

\section{CHARACTERISTICS OF THE SAMPLE}

Files were examined from 87 children who were first fitted with hearing aids before 6 months of age. Four cases were excluded from further review as no data were available, leaving a sample size of 83 children. Of these 83 children, $5(6 \%)$ were identified as Aboriginal or Torres Strait Islander. Sixty-four children (77\%) had bilateral hearing aid fittings.

\section{TIMING OF CORTICAL AUDITORY EVOKED POTENTIALS WITHIN THE INITIAL FITTING PROGRAM}

Of the 83 children included in the file review, 55 children $(66 \%)$ had at least one CAEP assessment completed. Of these 55 children, 53 had CAEP assessment using HEARLab during the initial fitting program. Two children had their initial CAEP assessment prior to amplification because they were diagnosed 
with ANSD, and CAEPs were used as part of a battery of tests to ascertain if they required amplification. Both of these children were identified as having absent responses to some or all speech sounds tested and were subsequently fitted with hearing aids within 2 to 3 weeks of their CAEP assessment. Fig. 3 shows that of those 55 children who had CAEPs to evaluate their hearing aid fitting, $35(64 \%)$ had the assessment within 8 weeks of their fitting.

\section{NUMBER OF CORTICAL AUDITORY EVOKED POTENTIAL APPOINTMENTS}

Of the 55 children who were CAEP tested during their initial fitting program, Fig. 4 shows the number of CAEP appointments that were conducted for each individual child. Repeat testing usually followed the audiogram being reestimated and hearing aids being adjusted based on the initial CAEP assessment. Subsequently further assessment(s) were performed to evaluate the effectiveness of the new aid adjustments.

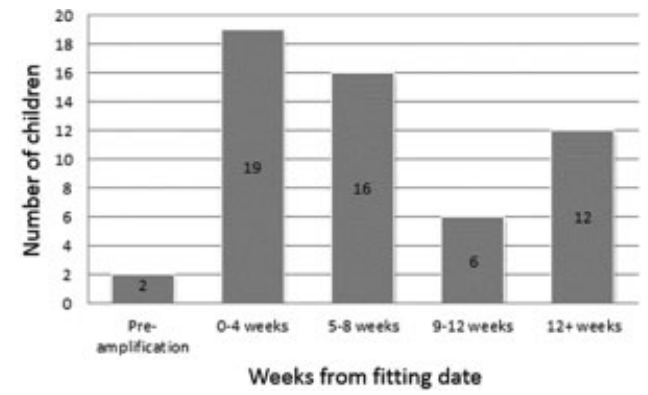

Figure 3 Interval between fitting and first cortical auditory evoked potential assessment.

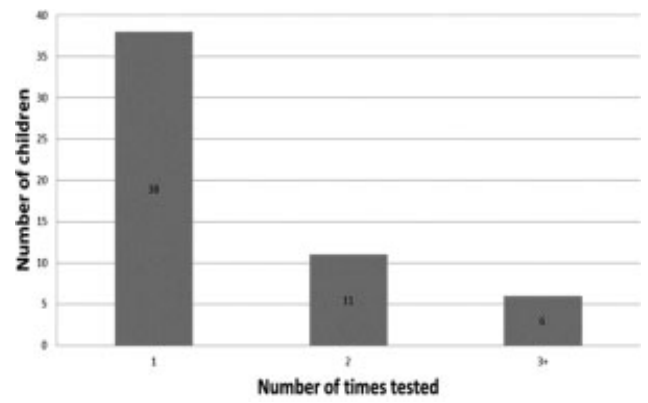

Figure 4 How often cortical auditory evoked potentials were used in infant fitting programs.
Thirty-eight children (69\%) were only assessed once with HEARLab, although optimally children should come in for a second CAEP appointment to verify a modified hearing aid fitting. More often, once the fitting, follow-up, CAEP, and aid adjustment were completed, the child was old enough to complete behavioral testing. When behavioral information becomes available, more emphasis is placed on fitting to a prescription based on the behavioral audiogram obtained using Visual Reinforcement Orientation Audiometry (VROA). Of the six children (11\%) who had two or more HEARLab assessments, one child had more than four assessments over a period of 5 months because of a deteriorating hearing loss that required frequent aid adjustment and evaluation via CAEP testing. This child went on to be implanted, a decision supported by the fact that consistent aided CAEPs could not be achieved. In three cases, the child attended but did not complete the assessment because the child fell asleep or was not in the ideal state for testing. If this occurs, typically a new appointment is booked as soon as possible.

\section{REASONS CORTICAL AUDITORY EVOKED POTENTIAL ASSESSMENT WAS NOT PERFORMED}

Of the 83 children included in the file review, 28 children (33\%) did not receive a CAEP assessment. Fig. 5 shows the reasons CAEP testing was not used to evaluate fittings. Fifteen

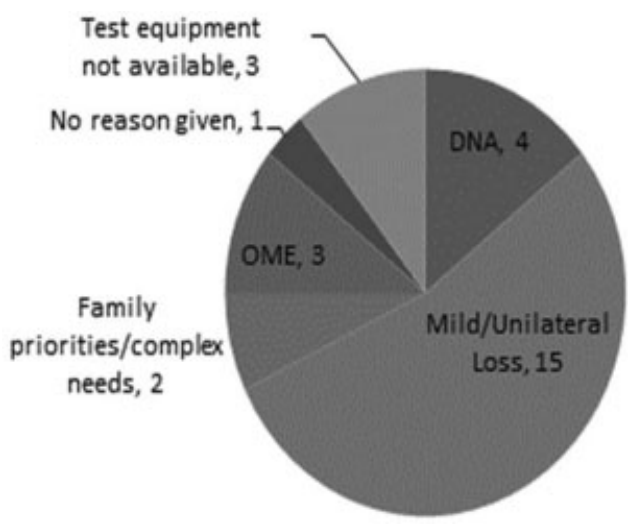

Figure 5 Reasons why cortical auditory evoked potential assessment was not performed in the infant fitting program $(n=28)$. Abbreviations: DNA, did not attend; OME, otitis media effusion. 
of the aided children had a mild or unilateral hearing loss. Thus according to Australian Hearing protocol, they were not prioritized for aided CAEP assessment.

This means that the remaining 68 children were eligible for CAEP testing. Of these 68 children, 13 (19\%) did not get tested with CAEPs, although they should have been according to the Australian Hearing CAEP protocol. Four children failed to attend the scheduled CAEP appointment. Equipment was unavailable in three cases. Three children had otitis media effusion, which prevented testing. Two children were fitted with hearing aids at close to 6 months of age due to other family priorities. It was hence possible to assess them using VROA within 2 months of fitting. Because reliable behavioral thresholds are preferred for deriving a hearing aid prescription, the clinical program for these two children changed focus from objective CAEP assessment to behavioral assessment. In one case, no valid reason was given.

Of the reasons stated above, only the last case $(2 \%)$ possibly was not tested because the protocol was not followed correctly.

\section{Audiologist Survey}

\section{CORTICAL AUDITORY EVOKED POTENTIAL EXPERIENCE}

Of the 32 of 48 clinicians who responded (a $67 \%$ response rate), 18 (56\%) had conducted CAEP testing for more than 2 years in their clinic. Eight $(25 \%)$ had performed CAEP evaluations for less than 6 months. Two (6\%) had performed more than 30 CAEP assessments in the last 12 months. Sixteen (50\%) had tested between 10 and 30 clients in the last year.

\section{CLIENTS TESTED}

Fig. 6 shows the distribution of the ages of clients who had been tested at least once by a pediatric clinician (multiple answers were possible). As can be observed from Fig. 6, all but one clinician (97\%) had tested the target age group, which is infants fitted at $<6$ months of age. At this age, generally no reliable behavioral testing can be obtained.

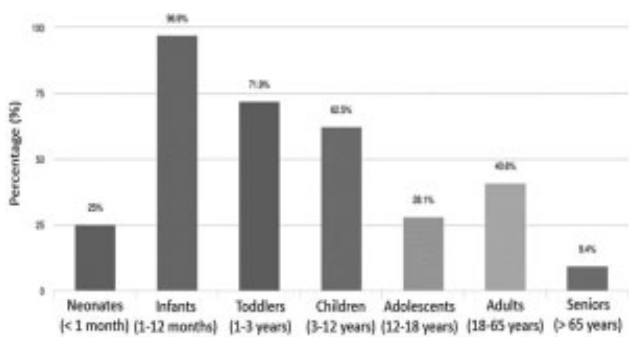

Figure 6 Client groups/patients assessed using the HEARLab system, Frye Electronics, Tigard, Oregon.

\section{APPOINTMENT LENGTH}

A complete appointment (including impressions, hearing aid check, setting up the equipment, settling the child, performing the CAEP test, explaining the results, and hearing aid fitting adjustment) generally took 60 minutes or less (indicated by 23 or 72\%); 12 clinicians $(38 \%)$ indicated that testing lasted 45 minutes or less, and 6 (19\%) indicated 90 minutes or more. This shows that testing time can vary considerably. Test time may be influenced by the experience of the clinician, the availability of a second clinician to assist and distract the child, the protocol used (number of stimuli used and intensity levels chosen), and the state of arousal of the child. The last reason was perceived by the audiologist as the most likely reason for such a large variability in appointment times. The majority ( 23 or $72 \%$ ) of clinicians tested at two stimulus presentation levels, as recommended by the flowchart in Fig. 2.

\section{SATISFACTION WITH CORTICAL AUDITORY EVOKED POTENTIAL ASSESSMENT}

Table 1 presents answers to six statements related to interpretation, reception, and satisfaction with CAEP testing. The majority of the users agreed with the statements. The clinicians were also invited to provide voluntary comments to statements 1 to 4 in Table 1 , and to these three questions:

- How do you predominantly use the information provided by the system?

- Can you provide two examples where you think cortical testing was not useful?

- Can you provide two examples where you think cortical testing was useful? 
Table 1 Additional Statements from the HEARLab, Frye Electronics, Tigard, Oregon, User Survey

\begin{tabular}{|c|c|c|c|c|c|}
\hline Statements & $\begin{array}{l}\text { Strongly } \\
\text { Disagree }\end{array}$ & Disagree & Neutral & Agree & $\begin{array}{l}\text { Strongly } \\
\text { Agree }\end{array}$ \\
\hline $\begin{array}{l}\text { 1. The CAEP results influence my approach } \\
\text { to rehabilitation. }\end{array}$ & $1(3 \%)$ & $0(0 \%)$ & $0(0 \%)$ & $21(66 \%)$ & $10(31 \%)$ \\
\hline $\begin{array}{l}\text { 2. The CAEP results are clinically consistent } \\
\text { with behavioral results, where applicable. }\end{array}$ & $0(0 \%)$ & $0(0 \%)$ & $3(9 \%)$ & $24(75 \%)$ & $5(16 \%)$ \\
\hline 3. The CAEP results are easy to interpret. & $0(0 \%)$ & $1(3 \%)$ & $1(3 \%)$ & $21(66 \%)$ & $9(28 \%)$ \\
\hline $\begin{array}{l}\text { 4. The CAEP results are positively received } \\
\text { by parents. }\end{array}$ & $1(3 \%)$ & $0(0 \%)$ & $2(6 \%)$ & $21(66 \%)$ & $8(25 \%)$ \\
\hline $\begin{array}{l}\text { 5. I would recommend CAEP testing to } \\
\text { other Audiologists who do not have the } \\
\text { HEARLab system. }\end{array}$ & $1(3 \%)$ & $0(0 \%)$ & $0(0 \%)$ & $16(50 \%)$ & $15(47 \%)$ \\
\hline $\begin{array}{l}\text { 6. Overall, I am satisfied with CAEP testing } \\
\text { (using the HEARLab system)? }\end{array}$ & $0(0 \%)$ & $0(0 \%)$ & $0(0 \%)$ & $21(68 \%)$ & $10(32 \%)$ \\
\hline
\end{tabular}

Abbreviation: CAEP, cortical auditory evoked potential.

In response to the four statements and three questions, the clinicians noted that (in descending order of occurrence):

- The results were used to verify or modify hearing aid fittings (27 of 30 clinicians who left comments).

- CAEPs were used for counseling parents to reinforce the need for a hearing aid, or to reassure them that the hearing aid was providing sufficient amplification (17 of 30).

- If no aided CAEPs were observed with multiple visits, CAEP testing provided additional information for the decision process toward cochlear implantation. Conversely, if CAEPs were observed when none were expected, the decision toward cochlear implantation was delayed until more (behavioral) information became available (17 of 30).

- The results were used in conjunction with other information (e.g., parent feedback, the $\mathrm{PEACH},{ }^{44}$ and the estimated audiogram based on electrophysiologic measures; 13 of 30).

- The results provided additional information on unaided ability in ANSD cases (12 of 30).

- The results provided earlier reassurance than when using behavioral paradigms that speech was being detected with the hearing aids (10 of 30).
- It can be useful for clients who cannot provide reliable behavioral feedback (e.g., with developmental delays; 9 of 30).

- Occasionally CAEPs were not detected when it was believed that the sound was audible, given the estimated audiogram and despite testing under optimal conditions (low electroencephalogram noise, good impedance, child alert and settled, tympanograms consistent with normal middle ear function, and hearing aids matched to target). It has been shown that nondetected CAEPs are a possibility in young children. $^{8-11}$ Conversely, CAEPs were occasionally detected although it was believed that the sound was inaudible, a inevitable consequence of using a statistical paradigm. ${ }^{15,20}$ This highlights the need to combine CAEP results with other available information to provide an overall picture and, importantly, to avoid confusing families (7 of 30).

- Parents found it hard to relate CAEP results with the (aided) audiogram (4 of 30).

- Statistical results were sometimes difficult to interpret. For example, statistical presence of a response to a specific speech sound at a lower level occasionally coincided with the statistical absence at a higher level (3 of 30).

- Parents became frustrated if results were inconclusive or not matching expectations (1 of 30$)$. 


\section{DIFFICULTIES WITH TEST PROCEDURE}

According to the clinicians, CAEP testing was occasionally not clinically possible or difficult (in descending order of occurrence):

- Children with a noisy electroencephalogram were difficult or impossible to test. Noise was related to child behavior (e.g., movement), additional disabilities, or seizures (10 of 30 clinicians who left comments).

- Technical malfunctions with the recording system could cause issues as well. Examples include broken (electrode) cables, high electrode impedances, and software issues (1 of 30).

- Parents were against the use of the test and preferred to wait until behavioral responses were available (1 of 30$)$.

\section{DETAILED CASE STUDIES}

Three detailed case studies were selected to illustrate how CAEP testing can be used in a clinical environment.

\section{Case Study 1: Cochlear Implant Candidacy}

This child was referred to Australian Hearing via the UNHS program. Table 2 shows the tone burst ABR test results for the four audiometric frequencies. There were no responses at $95 \mathrm{~dB}$ normal hearing level (nHL) at all test frequencies. Based on the corrections suggested by Vander Werff et al, ${ }^{39}$ an estimated behavioral audiogram was derived (Table 3). According to the procedure described by Vander Werff et $\mathrm{al},{ }^{39}$ the threshold for $500-\mathrm{Hz}$ tone burst

Table 2 Case Study 1: Tone-burst auditory brainstem response (ABR) test results at diagnosis, in decibels of normal hearing level (dB nHL)

\begin{tabular}{lllll}
\hline & \multicolumn{4}{l}{ Frequency (Hz) } \\
\cline { 2 - 5 } & $\mathbf{5 0 0}$ & $\mathbf{1 , 0 0 0}$ & $\mathbf{2 , 0 0 0}$ & $\mathbf{4 , 0 0 0}$ \\
\hline Right & $>95$ & $>95$ & $>95$ & $>95$ \\
Left & $>95$ & $>95$ & $>95$ & $>95$ \\
\hline
\end{tabular}

Note: Tone burst auditory brainstem response test results at diagnosis (in decibels of normal hearing level [nHL]). For conversion purposes, $>95 \mathrm{~dB} \mathrm{nHL}$ is taken equal to $100 \mathrm{~dB} \mathrm{nHL}$, a lower limit of the estimated hearing loss.
Table 3 Case Study 1: Estimated audiogram at diagnosis, in decibels of hearing level (dB HL)

\begin{tabular}{lllll}
\hline & \multicolumn{4}{l}{ Frequency (Hz) } \\
\cline { 2 - 5 } & $\mathbf{5 0 0}$ & $\mathbf{1 , 0 0 0}$ & $\mathbf{2 , 0 0 0}$ & $\mathbf{4 , 0 0 0}$ \\
\hline Right & 90 & 95 & 100 & 105 \\
Left & 90 & 95 & 100 & 105 \\
\hline
\end{tabular}

Resulting estimated audiogram (in decibels of hearing level $(\mathrm{HL})$ using decibels of normal hearing level $(\mathrm{nHL})$ to decibels of $\mathrm{HL}$ correction, derived from Vander Werff et al. ${ }^{39}$ The values in Table 2 are corrected by $-10,-5$, 0 , and $+5 \mathrm{~dB}$ for frequencies $0.5,1,2$, and $4 \mathrm{kHz}$,

respectively, to convert from decibels of $\mathrm{nHL}$ to decibels of $\mathrm{HL}$.

(in decibels of nHL) had to be corrected (improved) by $10 \mathrm{~dB}$ to provide an estimated behavioral threshold (in decibels of HL). Hence, the ABR threshold for $500 \mathrm{~Hz}$ in Table 2, which was at least $100 \mathrm{~dB}$ nHL, was corrected to $90 \mathrm{~dB}$ HL. Similarly, 1, 2, and $4 \mathrm{kHz}$ were corrected by $-5,0$ and $+5 \mathrm{~dB}$, respectively, to convert from decibels of nHL to decibels of HL. Typically a child with ABR results suggesting a severe or profound hearing loss will be offered a simultaneous referral to a CI agency along with a referral to Australian Hearing. This family, however, wished to defer CI agency referral until further information from a hearing aid trial indicated whether or not the child had aided access to speech sounds.

The child was fitted with hearing aids at 6 weeks of age based on the estimated behavioral audiogram in Table 3. Initial aided CAEP testing was conducted 2 weeks later at the first follow-up appointment. Fig. 7 shows the grand averages of the CAEPs to three speech stimuli $(/ \mathrm{m} /, / \mathrm{g} /$, and $/ \mathrm{t} /)$ at 65 and $75 \mathrm{~dB}$ SPL via a loudspeaker when the child was bilaterally aided. The figure also shows the associated $p$ values of the statistical test incorporated in the HEARLab system. A $p$ value of 0.05 or smaller indicates a high likelihood of a CAEP being present. No CAEPs were detected for any speech sounds at all tested intensities. In accordance with Australian Hearing protocols, the estimated audiogram was assumed to be $1.5 \mathrm{SD}$ (or $15 \mathrm{~dB}$ worse) than originally estimated at all frequencies. The hearing aids were then adjusted to meet NAL-NL2 targets at 50, 65, and $80 \mathrm{~dB}$ SPL inputs for this new estimated audiogram (see Table 4). 

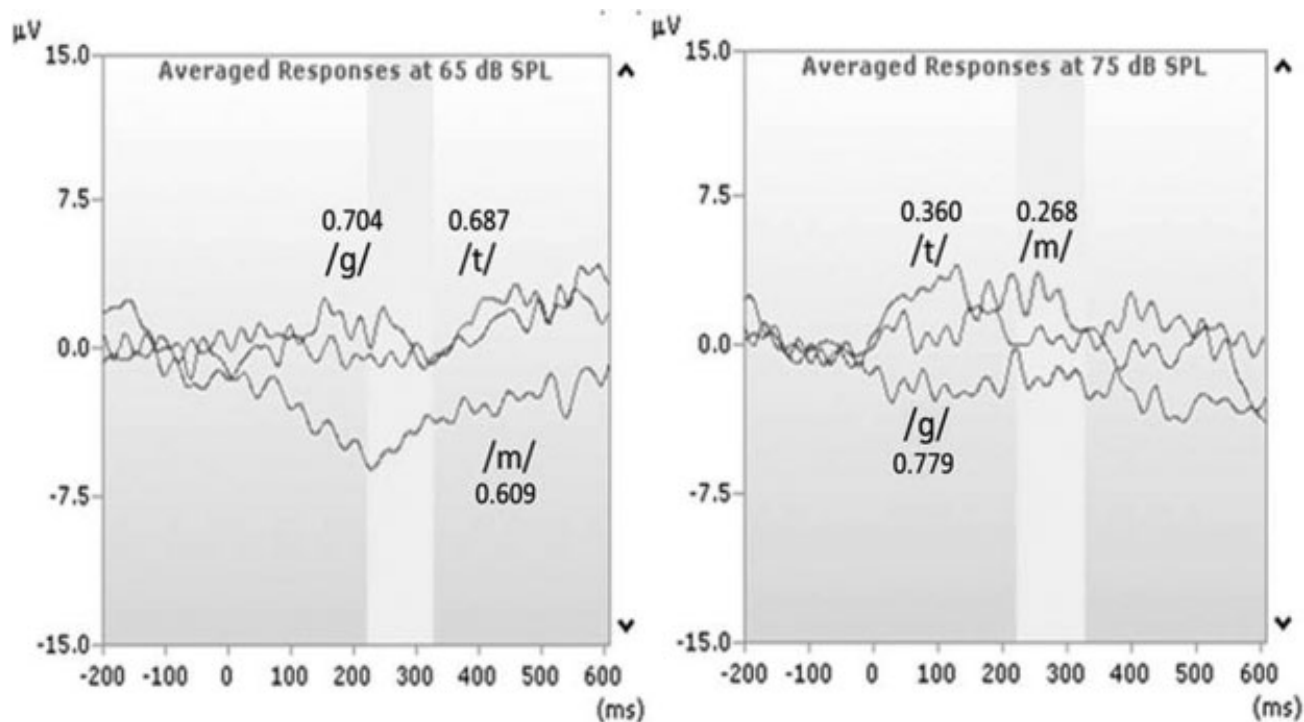

Figure 7 Case study 1: Cl candidacy. Absent responses to speech stimuli /m/, /g/, and /t/ at 65 and $75 \mathrm{~dB}$ sound pressure level (SPL) via a loudspeaker when bilaterally aided. Statistical $p$ values for every speech sound are provided ( $p \leq 0.05$ indicates a high likelihood of a cortical auditory evoked potential being present).

The child was subsequently reassessed at the age of 3 months using aided CAEPs in the free field while bilaterally aided with inputs of 65 and $75 \mathrm{~dB}$ SPL for all three speech sounds. Again, $p$ values shown in Table 5 were not significant at either conversational (65 dB SPL) or elevated (75 dB SPL) speech levels. This suggested at least a profound hearing loss and little or no benefit from hearing aids. Results were explained to the parents, and CI agency referral was suggested as the next step in this

Table 4 Case Study 1: Estimated audiogram, in decibels of hearing level ( $\mathrm{dB} \mathrm{HL}$ ), after adjustment based on cortical response testing

\begin{tabular}{lllll}
\hline & \multicolumn{4}{l}{ Frequency $(\mathbf{H z})$} \\
\cline { 2 - 5 } & $\mathbf{5 0 0}$ & $\mathbf{1 , 0 0 0}$ & $\mathbf{2 , 0 0 0}$ & $\mathbf{4 , 0 0 0}$ \\
\hline Right & 105 & 110 & 115 & 120 \\
Left & 105 & 110 & 115 & 120 \\
\hline
\end{tabular}

Table 5 Case Study 1: Statistical p-values of cortical responses

\begin{tabular}{llll}
\hline & /m/ & /g/ & /t/ \\
\hline 75 dB SPL & 0.617 & 0.418 & 0.705 \\
$65 \mathrm{~dB} \mathrm{SPL}$ & 0.639 & 0.505 & 0.318
\end{tabular}

Abbreviation: SPL, sound pressure level.

Note: Results are statistical $p$ values for the aided three speech sounds at two stimulus levels. child's program management. Reports on these results were sent to the family's early intervention service and CI center, where further testing was conducted. Eventually, the child went on to receive bilateral CIs at 5 months of age. The child's parents e-mailed the audiologists to say, "Thank you so much for the information you gave us on the previous testing as it helped us with our decision to proceed with the implants."

\section{Case Study 2: Ensuring Audibility}

This child was referred to Australian Hearing via the UNHS program. Diagnostic ABR results (Table 6) were used to estimate a bilateral mild sloping to moderately severe sensorineural hearing loss (Table 7). The child was fitted with

Table 6 Case Study 2: Tone Burst Auditory Brainstem Response Testing Results at Diagnosis (in Decibels of normal hearing level [nHL])

\begin{tabular}{lllll}
\hline & \multicolumn{4}{l}{ Frequency (Hz) } \\
\cline { 2 - 5 } & $\mathbf{5 0 0}$ & $\mathbf{1 , 0 0 0}$ & $\mathbf{2 , 0 0 0}$ & $\mathbf{4 , 0 0 0}$ \\
\hline Right & 40 & DNT & 60 & 70 \\
Left & 40 & DNT & 70 & 80 \\
\hline
\end{tabular}

Abbreviation: DNT, did not test.

Note: Thresholds at $1 \mathrm{kHz}$ are interpolated from the thresholds at 0.5 and $2 \mathrm{kHz}$. 
Table 7 Case Study 2: Resulting Estimated Behavioral Audiogram (in Decibels of $\mathrm{HL}$ ) Using Decibels of $n \mathrm{HL}$ to Decibels of $\mathrm{HL}$ Correction

\begin{tabular}{lllll}
\hline & \multicolumn{4}{l}{ Frequency (Hz) } \\
\cline { 2 - 5 } & $\mathbf{5 0 0}$ & $\mathbf{1 , 0 0 0}$ & $\mathbf{2 , 0 0 0}$ & $\mathbf{4 , 0 0 0}$ \\
\hline Right & 30 & 45 & 60 & 75 \\
Left & 30 & 50 & 70 & 85 \\
\hline
\end{tabular}

Abbreviations: $\mathrm{HL}, \mathrm{nHL}$, normal hearing level. Note: Derived from Vander Werff et al. ${ }^{39}$

bilateral hearing aids at 5 weeks of age based on the estimated hearing thresholds.

Aided CAEP testing was conducted in the free field at 4.5 months of age. Fig. 8 shows the CAEPs at 65 and $55 \mathrm{~dB}$ SPL of speech sounds $/ \mathrm{m} /, \mathrm{g} /$, and $/ \mathrm{t} /$ and the $p$ values associated with the different waveforms. At $65 \mathrm{~dB}$ SPL, all speech sounds evoked a CAEP, suggesting audibility across the speech frequency spectrum. According to the child's estimated audiogram (Table 7) and the NAL-NL2 speecho-gram (dark gray highlighted areas in Fig. 9), audibility was expected for low-, mid-, and high-frequency speech sounds at $55 \mathrm{~dB}$ SPL. Although the speech-o-gram only indicates predicted audibility for $50 \mathrm{~dB}$ SPL presentation levels, one can assume that if audibility is expected at $50 \mathrm{~dB}$ SPL then it is also expected at $55 \mathrm{~dB}$ SPL. As shown in Fig. 8, CAEPs at 55 $\mathrm{dB}$ SPL indicated audibility only for speech sound / $\mathrm{t} /$. Australian Hearing protocols determined that the audiogram should be reestimated to be $1 \mathrm{SD}(10 \mathrm{~dB})$ worse for low- and midfrequency speech sounds. The hearing aids were consequently adjusted to meet the NALNL2 targets for $50 \mathrm{~dB}$ SPL input.

A subsequent follow up was conducted 1 month later at 5.5 months of age. The CAEP waveforms and $p$ values (see Fig. 10) suggested that there was now good audibility for all speech sounds at conversational $(65 \mathrm{~dB}$ $\mathrm{SPL}$ ) and for $/ \mathrm{g} / \mathrm{and} / \mathrm{t} / \mathrm{at}$ softer levels $(55 \mathrm{~dB}$ SPL). Typically when the gain of a hearing aid is increased, the audiologist will conduct an informal test of loudness tolerance or "maximum power output check" via moderate- and loud-intensity impulse sounds to ensure the child is not overamplified. No tolerance issues were detected to impulse sounds in the clinic setting via maximum power output check, and the child's parents reported no discomfort to sounds in everyday listening environments. The parents were reassured about appropriateness of hearing aid settings and a resulting e-mail was sent by the mother to the audiologists where she
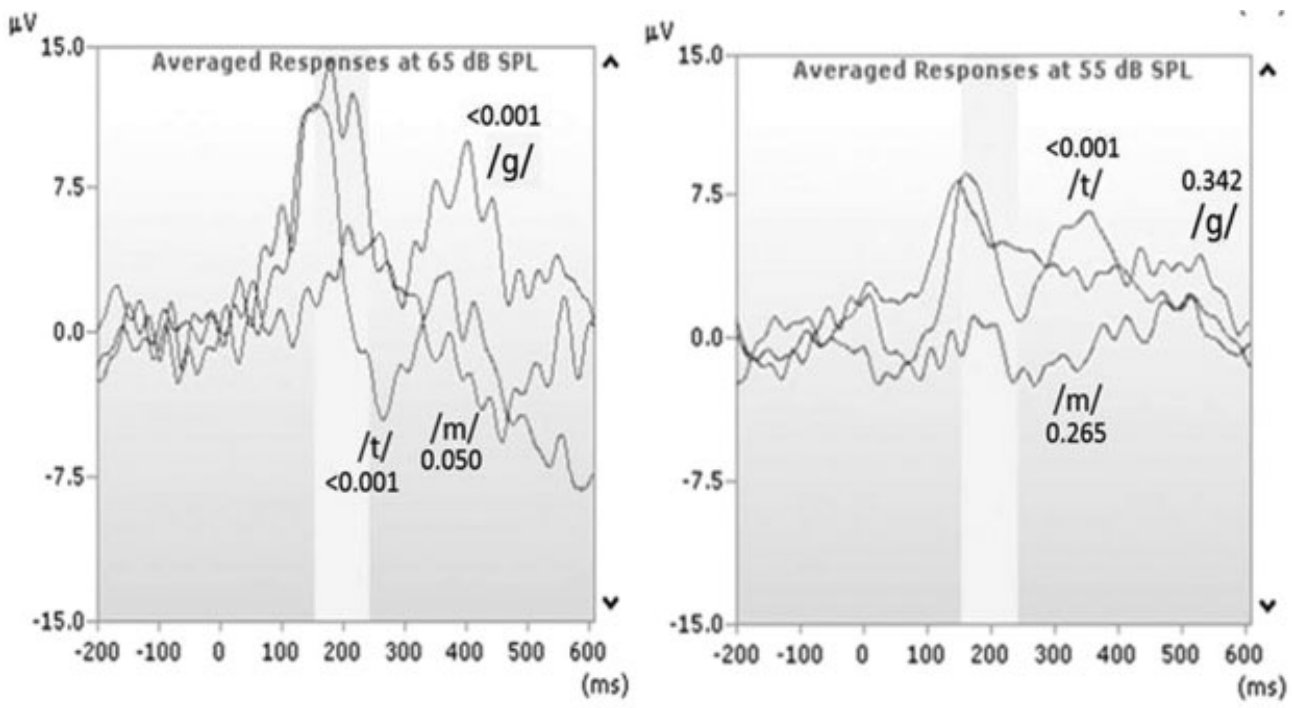

Figure 8 Case study 2: first visit. Present responses to speech stimuli /m/, /g/, and /t/ at $65 \mathrm{~dB}$ sound pressure level (SPL) and to the /t/ stimulus at $55 \mathrm{~dB}$ SPL via a loudspeaker when bilaterally aided. Statistical $p$ values for every speech sound are provided ( $p \leq 0.05$ indicates a high likelihood of a cortical auditory evoked potential being present). 


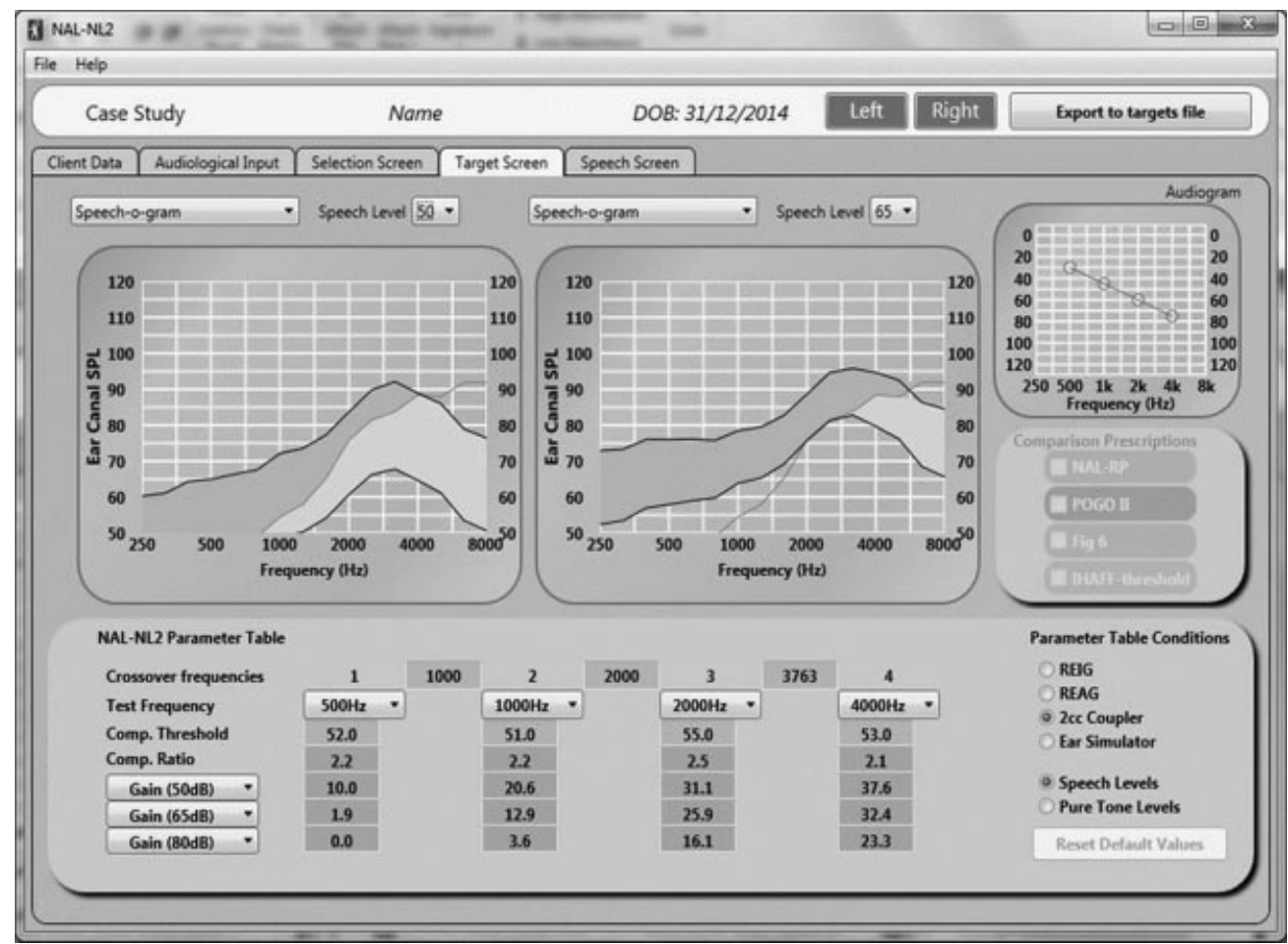

Figure 9 Case study 2: Expected audibility for 50 and $65 \mathrm{~dB}$ sound pressure level speech as indicated by NAL-NL2 speech-o-gram.
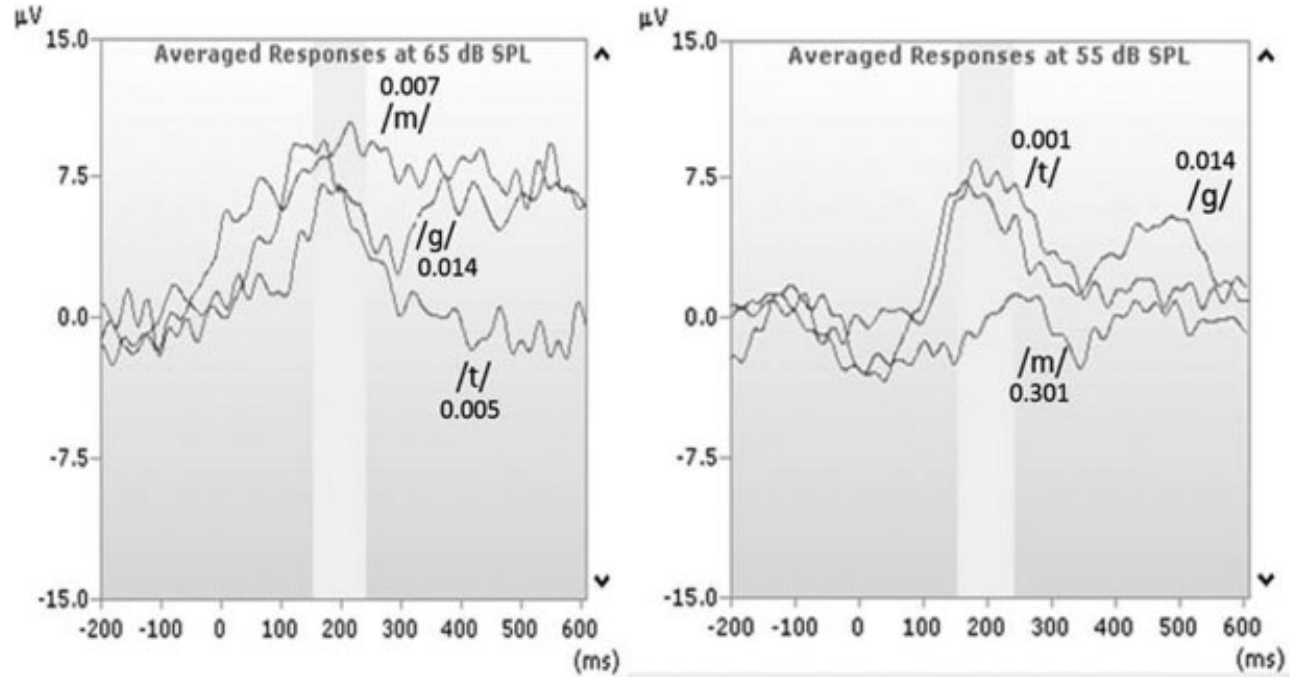

Figure 10 Case study 2: second visit. Present responses to speech stimuli /m/, /g/, and /t/ at $65 \mathrm{~dB}$ sound pressure level (SPL) and to the /g/ and /t/ stimuli at $55 \mathrm{~dB}$ SPL via a loudspeaker when bilaterally aided. Statistical $p$ values for every speech sound are provided ( $p \leq 0.05$ indicates a high likelihood of a cortical auditory evoked potential being present).

commented: "We feel very relieved, as our faith has been restored in the hearing aids as a result of what we discovered from the results."

\section{Case Study 3: Decision to Fit Hearing} Aids in Case of ANSD

This child was referred to Australian Hearing via the UNHS program. Diagnostic audiology 
assessment showed clear cochlear microphonics on reversal of click stimulus polarity during $\mathrm{ABR}$ and distortion product otoacoustic emissions were present in both ears, indicating ANSD.

Unaided CAEP testing in the free field was conducted at Australian Hearing at the age of 3 months. Fig. 11 shows responses to (and corresponding $p$ values of) all three speech sounds $(/ \mathrm{m} /, / \mathrm{g} /$, and $/ \mathrm{t} /)$ at $65 \mathrm{~dB} \mathrm{SPL}$. Responses also were obtained at $55 \mathrm{~dB}$ SPL for $/ \mathrm{g} /$ and $/ \mathrm{t} /$, but were inconclusive for $/ \mathrm{m} /$ due to high noise levels. Although there appeared to be a possible waveform in response to the $/ \mathrm{m} /$ stimuli, this may be caused by the background noise. A PEACH diary, ${ }^{44}$ which records the parents' observations of hearing responses in daily environments over a period of time, was given to the child's parents to complete. A retest of speech sound $/ \mathrm{m} /$ at $55 \mathrm{~dB}$ SPL was planned 1 month later.

At the age of 4.5 months, the child had significant responses to $/ \mathrm{m} /$ at $55 \mathrm{~dB}$ SPL, as shown in Fig. 12. The PEACH diary also was scored and results indicated that the child's

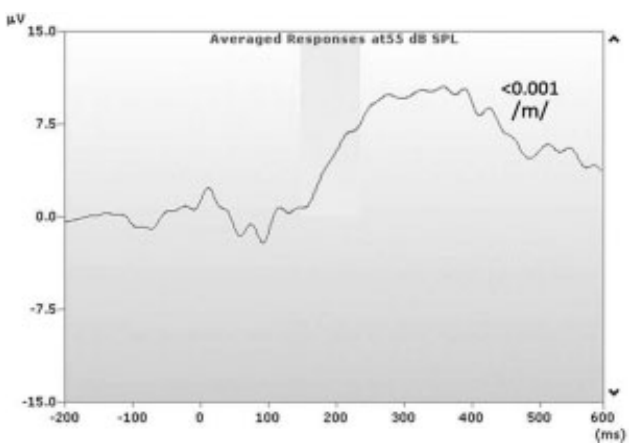

Figure 12 Case study 3: second visit. Present response at $55 \mathrm{~dB}$ sound pressure level (SPL) when testing $/ \mathrm{m} /$ only ( $p \leq 0.05$ ).

auditory function in everyday life situations was within $1 \mathrm{SD}$ of normally hearing children of the same age and developmental level.

The combined results suggested that the child was able to detect both conversational (65 dB SPL) and soft-level (55 dB SPL) speech sounds while unaided. This justified the earlier decision not to aid but to monitor the child's auditory responses via the PEACH diary and to conduct behavioral tests as soon as appropriate.
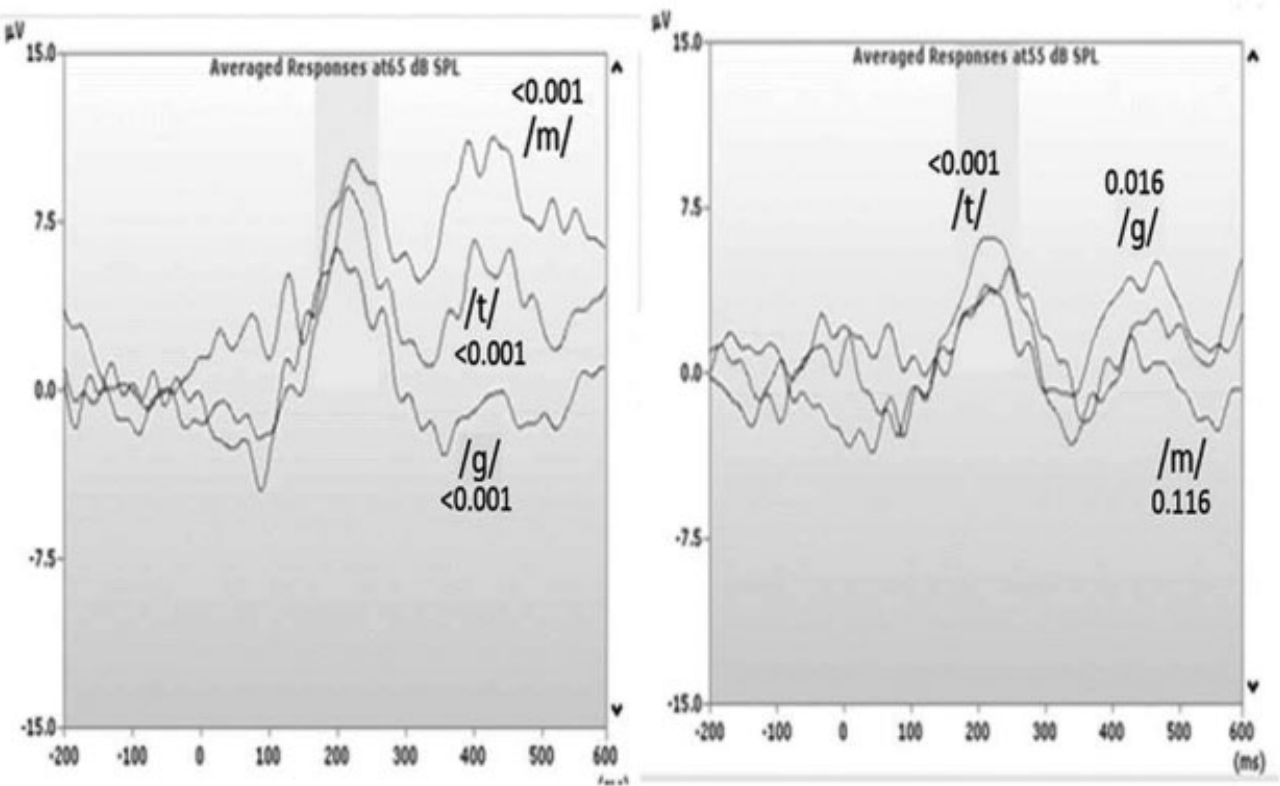

Figure 11 Case study 3: first visit. Present responses to speech stimuli /m/, /g/, and /t/ at $65 \mathrm{~dB}$ sound pressure level (SPL) and to the /g/ and /t/ stimuli at $55 \mathrm{~dB}$ SPL via a loudspeaker when bilaterally aided. Statistical $p$ values for every speech sound are provided ( $p \leq 0.05$ indicates a high likelihood of a cortical auditory evoked potential being present). 


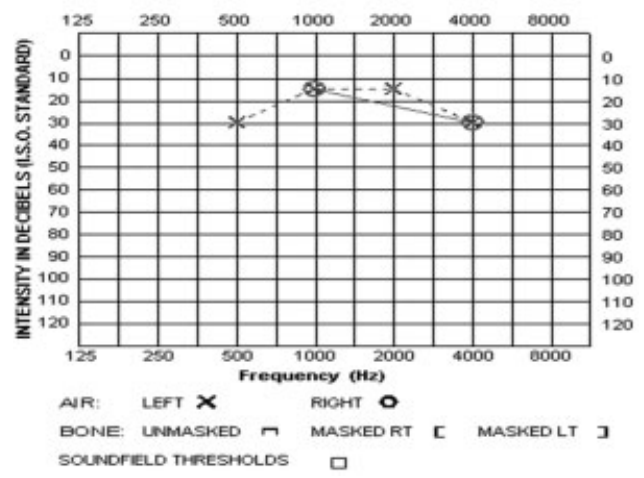

Figure 13 Case study 3: Latest audiogram obtained using play audiometry at the age of 2 years and 10 months. It is incomplete as testing had to be discontinued due to child's inattention.

VROA was not conducted until the child was 24 months old (Fig. 13) due to the presence of developmental delay and health problems. VROA results showed hearing thresholds within the normal range.

The child's parents reported no hearing difficulties at that time and amplification via hearing aids was deemed to have no or limited benefit. Monitoring continued via functional questionnaires, speech testing when appropriate, and behavioral hearing assessment. The family was advised to consider the use of a wireless remote microphone system if the child showed hearing difficulty in noise at a later stage. CAEP testing was useful in confirming the parents' initial observations of responses to soft sounds and in supporting the decision not to proceed with hearing aid fitting.

\section{DISCUSSION}

This article presented the clinical protocol for CAEP use that is currently being applied in the 25 Australian Hearing infant fitting centers across Australia. The file review of 68 cases eligible for CAEP testing (of 87 files) indicated that in $81 \%$ (55) of the cases, CAEP testing was performed when it was deemed necessary by the Australian Hearing CAEP protocol. This means that most children could be assessed in their initial fitting program when they were identified as a priority for CAEP testing. In $17 \%$ (12 of 68 ) of the cases, reasons for not testing were outside the control of the clinician. Only in $2 \%$ of the cases (1 of 68 ), the protocolpossibly-was not followed (with no valid reason provided). This shows that both the uptake of the new CAEP procedure and compliance with the Australian Hearing CAEP protocol is high. The review found that CAEP assessment is often achieved within a standard audiological appointment of up to 90 minutes. The aided CAEPs were most commonly tested within 8 weeks of the fitting. It is encouraging that there were few barriers (equipment being unavailable or the child not being in a good state of alertness for testing) to complete the test protocol. This indicates the feasibility of using CAEP testing in daily clinical practice.

Pediatric audiologists indicated that CAEP testing influenced their approach to rehabilitation and was well received by parents and that they were satisfied by the technique. Some audiologists reported, however, that parents were frustrated with inconclusive results or results that did not match expectations. This frustration can be addressed by appropriate explanation of the limitations of the testing and clear explanation of the next steps (e.g., CAEP retesting or a different assessment technique). Conversely, clinical experience shows as well that parents experience similar frustration when behavioral test results are inconclusive. The three case studies highlighted the four main situations where CAEP testing can be useful, more specifically to evaluate hearing aid fittings, indicate unaided audibility in ANSD cases, provide additional objective information when deciding on CI candidacy, and assist with parent counseling.

Overall, the review of the use of CAEPs in Australian Hearing clinics indicates that CAEPs can be effectively integrated into infant fitting programs and provide valuable information where behavioral testing is unreliable or impossible. Clinical verification of the CAEP testing method is still ongoing, however, and research questions remain. For example, to what extent does fine-tuning of a hearing aid fitting using the Australian Hearing clinical CAEP protocol result in a significant increase of CAEP presence at the second assessment? Does CAEP assessment contribute to a hearing 
aid fitting that is significantly closer to hearing aid target fitting than without? Do behavioral outcomes improve when CAEPs are used early to evaluate and adjust the hearing aid fitting? Finally, does CAEP assessment result in a significantly earlier decision to proceed with cochlear implantation?

\section{ACKNOWLEDGMENTS}

The authors would like to acknowledge HsiuWen Chang, Teresa Ching, Harvey Dillon, and Vicky Zhang for contributing to the draft of the clinical protocol on which this document is based. They also thank the clinicians who have completed the survey and provided valuable feedback. The authors acknowledge the financial support of the HEARing CRC, established and supported under the Cooperative Research Centres Program (an initiative of the Australian Government), the NAL, and the support of the Australian Government Department of Health.

\section{REFERENCES}

1. Bromley M, Wake M, Leigh G, et al. National framework for neonatal hearing screening. 2013: 1-67

2. Korczak PA, Kurtzberg D, Stapells DR. Effects of sensorineural hearing loss and personal hearing aids on cortical event-related potential and behavioral measures of speech-sound processing. Ear Hear 2005;26(2):165-185

3. Kurtzberg D, Stapells DR, Wallace IF. Eventrelated potential assessment of auditory system integrity: Implications for language development. In: Vietze PM, Vaughan HG, eds. Early Identification of Infants with Developmental Disabilities. New York, NY: Grune \& Stratton; 1988:160-180

4. Kraus N, McGee TJ, Koch DB. Speech sound representation, perception, and plasticity: a neurophysiologic perceptive. Audiol Neurootol 1998; 3(2-3):168-182

5. Dillon H. So, baby, how does it sound? Cortical assessment of infants with hearing aids. Hear J 2005;58(10):10-17

6. Pearce W, Golding M, Dillon H. Cortical auditory evoked potentials in the assessment of auditory neuropathy: two case studies. J Am Acad Audiol 2007;18(5):380-390

7. Rance G, Cone-Wesson B, Wunderlich J, Dowell R. Speech perception and cortical event related potentials in children with auditory neuropathy. Ear Hear 2002;23(3):239-253

8. Cone B, Whitaker R. Dynamics of infant cortical auditory evoked potentials (CAEPs) for tone and speech tokens. Int $\mathrm{J}$ Pediatr Otorhinolaryngol 2013;77(7):1162-1173

9. Van Dun B, Carter L, Dillon H. Sensitivity of cortical auditory evoked potential detection for hearing-impaired infants in response to short speech sounds. Audiol Res 2012;2(e13):65-76

10. Chang HW, Dillon H, Carter L, van Dun B, Young ST. The relationship between cortical auditory evoked potential (CAEP) detection and estimated audibility in infants with sensorineural hearing loss. Int J Audiol 2012;51(9):663-670

11. Gardner-Berry K, Chang H, Ching TYC, Hou S. Detection rates of cortical auditory evoked potentials at different sensation levels in infants with sensory/ neural hearing loss and auditory neuropathy spectrum disorder. Semin Hear 2016;37(1): 53-61

12. Rapin I, Graziani LJ. Auditory-evoked responses in normal, brain-damaged, and deaf infants. Neurology 1967;17(9):881-894

13. Barnet $\mathrm{AB}$. EEG audiometry in children under three years of age. Acta Otolaryngol 1971;72(1):1-13

14. Almeqbel A. Speech-evoked cortical auditory responses in children with normal hearing. S Afr J Commun Disord 2013;60:38-43

15. Carter L, Golding M, Dillon H, Seymour J. The detection of infant cortical auditory evoked potentials (CAEPs) using statistical and visual detection techniques. J Am Acad Audiol 2010;21(5):347-356

16. Carter L, Dillon H, Seymour J, Seeto M, Van Dun B. Cortical auditory-evoked potentials (CAEPs) in adults in response to filtered speech stimuli. J Am Acad Audiol 2013;24(9):807-822

17. Durante AS, Wieselberg MB, Carvalho S, et al. Cortical auditory evoked potential: evaluation of speech detection in adult hearing aid users. Cordas 2014;26(5):367-373

18. Golding M, Purdy S, Sharma M, Dillon H. The effect of stimulus duration and inter-stimulus interval on cortical responses in infants. Aust $\mathrm{N} Z \mathrm{~J}$ Audiol 2006;28(2):122-136

19. Golding M, Pearce W, Seymour J, Cooper A, Ching T, Dillon H. The relationship between obligatory cortical auditory evoked potentials (CAEPs) and functional measures in young infants. J Am Acad Audiol 2007;18(2):117-125

20. Golding M, Dillon H, Seymour J, Carter L. The detection of adult cortical auditory evoked potentials (CAEPs) using an automated statistic and visual detection. Int J Audiol 2009;48(12):833-842

21. Purdy SC, Sharma M, Munro KJ, Morgan CLA. Stimulus level effects on speech-evoked obligatory cortical auditory evoked potentials in infants with 
normal hearing. Clin Neurophysiol 2013;124(3): 474-480

22. Van Dun B, Kania A, Dillon H. Cortical auditory evoked potentials in (un)aided normal-hearing and hearing-impaired adults. Semin Hear 2015; In press

23. Billings CJ. Uses and limitations of electrophysiology with hearing aids. Semin Hear 2013;34(4): 257-269

24. Billings CJ, Papesh MA, Penman TM, Baltzell LS, Gallun FJ. Clinical use of aided cortical auditory evoked potentials as a measure of physiological detection or physiological discrimination. Int $\mathrm{J}$ Otolaryngol 2012;2012:365752

25. Billings CJ, Tremblay KL, Miller CW. Aided cortical auditory evoked potentials in response to changes in hearing aid gain. Int J Audiol 2011; 50(7):459-467

26. Billings CJ, Tremblay KL, Souza PE, Binns MA. Effects of hearing aid amplification and stimulus intensity on cortical auditory evoked potentials. Audiol Neurootol 2007;12(4):234-246

27. Easwar V, Glista D, Purcell DW, Scollie SD. The effect of stimulus choice on cortical auditory evoked potentials (CAEP): consideration of speech segment positioning within naturally produced speech. Int J Audiol 2012;51(12):926-931

28. Easwar V, Glista D, Purcell DW, Scollie SD. Hearing aid processing changes tone burst onset: effect on cortical auditory evoked potentials in individuals with normal audiometric thresholds. Am J Audiol 2012;21(1):82-90

29. Easwar V, Purcell DW, Scollie SD. Electroacoustic comparison of hearing aid output of phonemes in running speech versus isolation: implications for aided cortical auditory evoked potentials testing. Int J Otolaryngol 2012;2012:518202

30. Jenstad LM, Marynewich S, Stapells DR. Slow cortical potentials and amplification-Part II: Acoustic measures. Int J Otolaryngol 2012; 2012:386542

31. Marynewich S, Jenstad LM, Stapells DR. Slow cortical potentials and amplification-Part I: N1-P2 measures. Int J Otolaryngol 2012; 2012:921513

32. Tremblay KL, Billings CJ, Friesen LM, Souza PE. Neural representation of amplified speech sounds. Ear Hear 2006;27(2):93-103

33. Tremblay KL, Kalstein L, Billings CJ, Souza PE. The neural representation of consonant-vowel transitions in adults who wear hearing aids. Trends Amplif 2006;10(3):155-162

34. King A, Carter L, Van Dun B, Zhang V, Pearce W, Ching TYC. Australian Hearing Aided Cortical Evoked Potentials Protocols. 2014; http://hearlab. nal.gov.au/practical-AH-protocol.shtml. Last accessed: 1 March 2015

35. Garinis AC, Cone-Wesson BK. Effects of stimulus level on cortical auditory event-related potentials evoked by speech. J Am Acad Audiol 2007;18(2): 107-116

36. King AM. The national protocol for paediatric amplification in Australia. Int J Audiol 2010;49 (Suppl 1):S64-S69

37. Martin BA, Tremblay KL, Stapells DR. Principles and applications of cortical auditory evoked potentials. In: Burkard RF, Eggermont JJ, Don M, eds. Auditory Evoked Potentials: Basic Principles and Clinical Application. Philadelphia, PA: Lippincott Williams and Wilkins; 2007:482-507

38. Sharma A, Martin K, Roland P, et al. P1 latency as a biomarker for central auditory development in children with hearing impairment. J Am Acad Audiol 2005;16(8):564-573

39. Vander Werff KR, Prieve BA, Georgantas LM. Infant air and bone conduction tone burst auditory brain stem responses for classification of hearing loss and the relationship to behavioral thresholds. Ear Hear 2009;30(3):350-368

40. King AM, Purdy SC, Dillon H, Sharma M, Pearce W. Australian Hearing protocols for the audiological management of infants who have auditory neuropathy. Aust N Z J Audiol 2005; 27(1):69-77

41. Dillon H. Hearing Aids. 2nd ed. New York, NY: Thieme Medical Publishers; 2012

42. Stapells DR, Gravel JS, Martin BA. Thresholds for auditory brain stem responses to tones in notched noise from infants and young children with normal hearing or sensorineural hearing loss. Ear Hear 1995;16(4):361-371

43. Bagatto MP, Scollie SD. Validation of the Parents' Evaluation of Aural/Oral Performance of Children (PEACH) Rating Scale. J Am Acad Audiol 2013; 24(2):121-125

44. Ching TYC, Hill M. The parents' evaluation of aural/oral performance of children (PEACH) scale: Normative data. J Am Acad Audiol 2007;18(3): 220-235 